\title{
CROSS-CORRELATION OF LARGE SCALE SURVEYS: RADIO-LOUD OBJECTS IN THE ROSAT ALL SKY SURVEY
}

\author{
W. BRINKMANN, W. YUAN, J. SIEBERT \\ MPI für Extraterrestrische Physik, Garching, FRG
}

\section{Introduction}

There are basically two different approaches to study the physical conditions and the energy transfer processes operating in astronomical objects:

- the detailed observations of a few prominent objects over a wide wavelength range.

- the study of the broad band properties of suitably choosen samples

Both approaches have some limitations when conclusions are drawn concerning the general (class) properties of different types of objects.

- Detailed observations of single sources give a maximum of information about that particular object. This is, however, in general 'well known'; i.e., near by, bright, well studied, and in many cases NOT AT ALL representative for its class.

- Samples of objects are usually rather small, selected from easily accessible sky regions, and biased towards the 'available instrument.'

We argue that a maximum of information about the typical qualities of various object classes can be gained by combining data obtained from large, sensitive, unbiased sky surveys.

We demonstrate this with some examples of radio-loud AGN, obtained from the correlation of the ROSAT All-Sky Survey with large scale radio surveys, like the $5 \mathrm{GHz}$ Green Bank Survey of the northern sky, providing a very large sample of more than 2000 radio-loud $\mathrm{X}$-ray sources. 


\section{X-ray versus Radio Properties}

Flux-ratio diagrams show a clear distinction between radio selected $\mathrm{BL}$ Lacs, X-ray selected BL Lacs, and quasars.

AGN's from the 87 GB-ROSAT sample (re-observed with the VLA) fall in the gap between the above 'extreme' type of objects (Brinkmann et al. 1996a). They lie near the position of radio-loud quasars shifted diagonally towards higher values of $f_{x} / f_{r}$ and $f_{o} / f_{r}$. This appears to be related to the fact that the average radio fluxes are generally about one order of magnitude lower than those of the previously known quasars.

Thus, the class of 'radio-loud' AGN covers a huge range in radio fluxes and this range is not bimodal but rather continuous. This could imply that the previously found gap in the flux ratio diagram was merely caused by selection effects as well and that radio-loud quasars (and, perhaps, BL Lacs) show a nearly linear relation in their flux ratios.

There have been claims that the X-ray spectral index of radio-loud quasars correlates with the radio spectral index and, eventually, with the core dominance and the radio loudness. The large number $(>600)$ of ROSAT detected RL QSOs seem to confirm these correlations. However:

- it remains unclear which physical quality is causing this correlation

- the behavior seems not to be continuous, but class dependent and bimodal.

The X-ray loudness $\alpha_{o x}=-0.384 \log \left(l_{2} \mathrm{keV} / l_{2500 \mathrm{~A}}\right)$ has been used frequently in the past for the discussion of the relative fraction of $\mathrm{X}$-ray to optical emission in an evolving quasar source population. There is no evolution with redshift but $\alpha_{o x}$ seems to depend on the quasars's optical luminosity. A regression analysis yields $\alpha_{o x} \sim 0.1 \times \log l_{o}$ with a probability level for a correlation of $P_{r}<10^{-8}$. However, at least a major fraction of the claimed correlation is caused by selection effects! In a simulation, assuming a constant $\alpha_{o x}$ with a certain dispersion as well as upper and lower luminosity limits for the X-ray data, the test sources occupy the same phase space region as the real data.

The boundaries of the distribution, causing the apparent correlation, became only visible from the large number of objects in the sample (Brinkmann et al. 1996b).

\section{References}

Brinkmann, W., et al. (1996a) Radio loud AGN in the ROSAT Survey, Astron.Astrophys. in press

Brinkmann, W., Yuan, W. and Siebert, J. (1996b) Broad band energy distribution of ROSAT detected quasars, Astron.Astrophys. in press 